\title{
DWI in Brains of Fetuses with Congenital Heart Disease: A Case-Control MR Imaging Study
}

\author{
(D).-Y. Ren, (D) H. Ji, (D). Zhu, and (D) S.-Z. Dong
}

\begin{abstract}
BACKGROUND AND PURPOSE: Abnormal ADC values are seen in ischemic brain lesions such as acute or chronic hypoxia. We aimed to assess whether ADC values in the developing brain measured by in utero DWI were different in fetuses with congenital heart disease compared with healthy controls.
\end{abstract}

MATERIALS AND METHODS: In utero DWI was performed in 50 fetuses with congenital heart disease and 100 healthy controls at a similar gestational age. Pair-wise ADC values of the ROls were manually delineated on each side of the frontal and periatrial WM and in the basal ganglia, thalamus, and cerebellar hemisphere, as well as a single measurement in the pons.

RESULTS: Fetuses with congenital heart disease had significantly lower ADC values in frontal and periatrial WM and the pons than controls (all $P<.05$ ) in the early stages of pregnancy. However, ADC values in the thalamus were higher for fetuses with congenital heart disease than for controls (gestational age, $\geq 26$ weeks). For $A D C$ values in the cerebellar hemisphere, there was no obvious significance between cases and controls $(P=.07)$ in the late stages of pregnancy. Basal ganglia ADC values were consistently not significantly different between the 2 groups during the early and late stages of pregnancy $(P=.47 ; .21)$.

CONCLUSIONS: Abnormal brain diffusivity can be detected using in utero DWI in fetuses with congenital heart disease. Abnormal ADC values found at a mean gestational age of 26 weeks suggest structural changes, which may provide an early indicator of the impact of congenital heart disease on the developing brain.

ABBREVIATIONS: CHD = congenital heart disease; GA = gestational age; HLHS = hypoplastic left-heart syndrome; TGA = transposition of the great arteries; TOF $=$ tetralogy of Fallot

C ongenital heart disease (CHD) is the most common congenital disability, affecting up about $0.6 \%-1.2 \%$ of live births and even more so in fetuses. ${ }^{1}$ Although most children with CHD can survive, neurologic impairment has been recognized as the most common complication of heart deficits after birth. ${ }^{2}$ Several studies have documented that certain congenital cardiac defects with altered circulation cause disturbances in brain oxygen and substrate deficiency,

Received March 23, 2021; accepted after revision June 23.

From the Department of Radiology, Shanghai Children's Medical Center, School of Medicine, Shanghai Jiao Tong University, Shanghai, P.R. China.

J.-Y. Ren and H. Ji contributed equally to this work.

This work was supported by the National Natural Science Foundation of China (81971582,81571628), Natural Science Foundation of Shanghai (19ZR1476700), and the Shanghai Pujiang Program (2019PJD030).

Abstract previously presented, in part, as an oral presentation at: Annual Meeting of the European Congress of Radiology, February 27 to March 3, 2019; Vienna, Austria.

Please address correspondence to Su-Zhen Dong, MD, PhD, Department of Radiology, Shanghai Children's Medical Center, School of Medicine, Shanghai Jiao Tong University, No.1678 Dong Fang Rd, Shanghai 200127; e-mail:

dongsuzhen@126.com

- Indicates open access to non-subscribers at www.ajnr.org

http://dx.doi.org/10.3174/ajnr.A7267 further reducing brain growth and maturation. In addition, these studies have indicated that fetuses with $\mathrm{CHD}$ may have delayed brain maturation in utero. ${ }^{3,4}$ Therefore, prenatal evaluation of the brain structure and function of fetuses with $\mathrm{CHD}$ is essential and may contribute to decision-making in the postnatal management.

DWI is MR imaging that uses the objective measurements of $\mathrm{ADC}$ values to detect water proton motion and diffusivity in cerebral tissues. Compared with the conventional MR imaging sequences, DWI is capable of detecting subtle alterations in brain diffusion associated with early brain hypoxic damage. ${ }^{5,6}$ Currently, fetal brain DWI has been used as a quantitative MR imaging method to assess the correlations of ADC with gestational age (GA) in various brain regions of healthy fetuses. However, it is not clear whether ADC could quantitatively evaluate early changes in brain development due to certain nonneurologic extra-CNS fetal pathologic conditions, especially for fetuses with $\mathrm{CHD}{ }^{7}$

This study aimed to investigate whether there were any differences in cerebral diffusion between fetuses with CHD and healthy controls using ADC values of in utero DWI in the early and late stages of pregnancy. 


\section{MATERIALS AND METHODS Overall Population}

Our study was authorized by the ethics commission of our hospital. All pregnant mothers involved in the study provided written informed consent before the examination for use of their clinical data for research purposes. All MR imaging studies were retrieved from our fetal MR imaging data base from August 2018 to June 2020. MR imaging was performed between 20 and 36 weeks of gestation (median GA, 26 weeks). GA was determined from the first day of the mother's last menstrual period and was confirmed by the results of an early second-trimester obstetric sonography. All fetal brain MR images were assessed by 2 pediatric radiologists (S.-Z.D. and M.Z.), who had 15 years of experience in fetal brain MR imaging, and a pediatric neuroradiologist (J.-Y.R.) with 3 years of experience in fetal brain MR imaging, to confirm a normal appearance. The fetuses with $\mathrm{CHD}$ were compared with a group of the healthy controls of similar GA. The subjects were subdivided on the basis of GA, with GA $<26$ weeks $(20-25.9$ weeks $)$ in one group and $\mathrm{GA} \geq 26$ weeks ( $26-36$ weeks) in a second group.

\section{Healthy Control Population}

The brain MR imaging of the fetuses that served as the control group were included after careful retrospective examination by the 2 experienced radiologists confirmed a normal brain appearance. Only singleton pregnancies eligible for fetal brain MR imaging in our hospital were included. Indications for fetal MR imaging consisted of extra-CNS abnormalities detected by sonography.

\section{CHD Study Population}

The cases of CHD were retrieved from the same fetal MR imaging data base. The inclusion criteria for retrieval were a single pregnancy and postnatally confirmed tetralogy of Fallot (TOF), double-outlet right ventricle, transposition of the great arteries (TGA), and hypoplastic left-heart syndrome (HLHS).

\section{Exclusion Criteria}

Exclusion criteria for the entire population were as follows: multiple pregnancies, fetal malformation or chromosomal abnormalities; associated arrhythmias; perinatal infection; fetal anemia; maternal conditions that might affect fetal hemodynamics such as pregestational diabetes, thyroid disease, or preeclampsia; any brain abnormality detected on conventional sequences; and cases in which DWI was not successfully performed or was nondiagnostic due to motion artifact degradation.

\section{MR Imaging Protocol}

All fetal brain MR imaging was performed using an Achieva 1.5T MR imaging scanner with a $60-\mathrm{mT} / \mathrm{m}$ gradient and a 16 -channel SENSE-XL-Torso coil (Philips Healthcare), and imaging included steady-state free precession, T2WI single-shot turbo spin-echo, T1WI, and DWI. The following parameters were used for the steady-state free precession: TR, $3.6 \mathrm{~ms}$; TE, $1.8 \mathrm{~ms}$; matrix, 216 $\times 218$; FOV, $260 \times 325 \mathrm{~mm}^{2}$; reverse corner, $80^{\circ}$; section thickness, 2-4 mm; spacing, $-2-0 \mathrm{~mm}$. The parameters for the single-shot turbo spin-echo sequence included TR/TE, 12,000/120 ms; matrix, $236 \times 220$; FOV, $260 \times 355 \mathrm{~mm}^{2}$; reverse angle, $90^{\circ}$; section thickness, $2 \mathrm{~mm}$ with 0 -mm spacing. The DWI sequence was performed in the transverse plane using b-values of 0 and $700 \mathrm{~mm}^{2} \mathrm{~s}^{-1}$. The maximal b-value of 700 was chosen to increase the SNR of the immature brain for demonstrating optimal contrast in the fetal brain. We used the following parameters: TR, $2494 \mathrm{~ms}$; TE, $96 \mathrm{~ms}$; section thickness, $4 \mathrm{~mm}$; FOV, $280 \times 320 \mathrm{~mm}^{2}$; matrix, $188 \times 125$; spacing, $0 \mathrm{~mm}$; flip angle, $90^{\circ}$. The scan time of the DWI sequence was 60 seconds. The total acquisition time was 15-25 minutes.

Pregnant women were in the supine or the left-sided position. No maternal or fetal sedation was used during the MR imaging examinations. First, the middle and lower abdomen of pregnant women was scanned in the coronal plane. This was followed by a focused multiplanar scan of the fetal brain. Subsequently, the fetal chest and abdomen were scanned in the axial, sagittal, and coronal planes. The repeat data acquisition or breath-holding of pregnant women at the end of expiration or both were used to reduce fetal motion artifacts to improve the success of the in utero DWI sequence.

\section{Imaging Analysis}

The DWI data were transmitted to a workstation (ADW4.4; GE Healthcare, USA). The ADC measurements were manually drawn in 11 circular, different ROIs. Pair-wise ADC measurements were obtained in the frontal and periatrial WM of each cerebral hemisphere and in the basal ganglia, thalami, and cerebellar hemispheres, as well as a single measurement in the pons. These measurements are based on ROI locations reported in previous literature. ${ }^{8}$ The ROIs were manually traced. They varied in size depending on the brain region and GA. The sizes of ROIs ranged from 20 to $60 \mathrm{~mm}^{2}$. The frontal WM ROIs were drawn anterior to the frontal horns on the inferior section. Both frontal WM and periatrial WM ROIs extended across the cerebral mantle, spanning the germinal matrix zone and the cortex, including the intermediate zone and the subplate. The periatrial WM ROIs were ovoid and placed midway between the anterior and posterior margins of the ventricular atrium. The width of the brain was multiplied by a constant factor, and the resulting products were used as the area of the periatrial WM ROIs. ${ }^{9}$ For each ROI, a mean ADC value (SD) $\left(10^{-3} \mathrm{~mm}^{2} / \mathrm{s}\right)$ was obtained. ADC values from both sides of the brain were averaged for each anatomic location described above. Examples of ROI positioning are shown in Fig 1.

Manual ADC measurements of all cases were performed by the same pediatric neuroradiologist with 3 years of fetal brain MR imaging experience (observer 1, J.-Y.R.) after a training session. A subgroup of 30 randomly selected subjects was re-analyzed by the same operator after a 2-month interval, blinded to the initial results, to investigate intraobserver reproducibility. A different subgroup of 30 fetal brains was analyzed by a pediatric radiologist with 15 years of fetal brain MR imaging experience (observer 2, S.-Z.D.) to study interobserver reliability.

\section{Statistical Analysis}

The first step of our delineation consisted of comparison of ADC values in the ROIs marked in each hemisphere (right versus left) using a Student paired $t$ test. The mean ADC value was presented graphically with quadratic curve fitting. For each ROI, the mean $\mathrm{ADC}$ value was plotted against the GA, and the relationship was assessed by linear regression between the CHD group and controls. Statistical comparisons between the control population and 
fetuses with CHD adjusted for GA were analyzed using the nonparametric Mann-Whitney $U$ test. The intraclass correlation coefficient was calculated to convey the association within and between observers for ADC measurements. A $P$ value $<.05$ was considered statistically significant. All analyses were calculated by SPSS 22.0 software (IBM).

\section{RESULTS}

\section{Study Cohort}

For the CHD group, we retrospectively identified 55 singleton pregnancies eligible for inclusion in our study, which fulfilled the inclusion criteria for the CHD group. Five of the 55 fetuses in $\mathrm{CHD}$ group were further excluded from this study because the anatomic structures on the DWI sequence were not clear ventricular system and clear brain parenchyma; to accurately measure the ADC values. The subtypes of the cardiac lesions are summarized in Table 1.
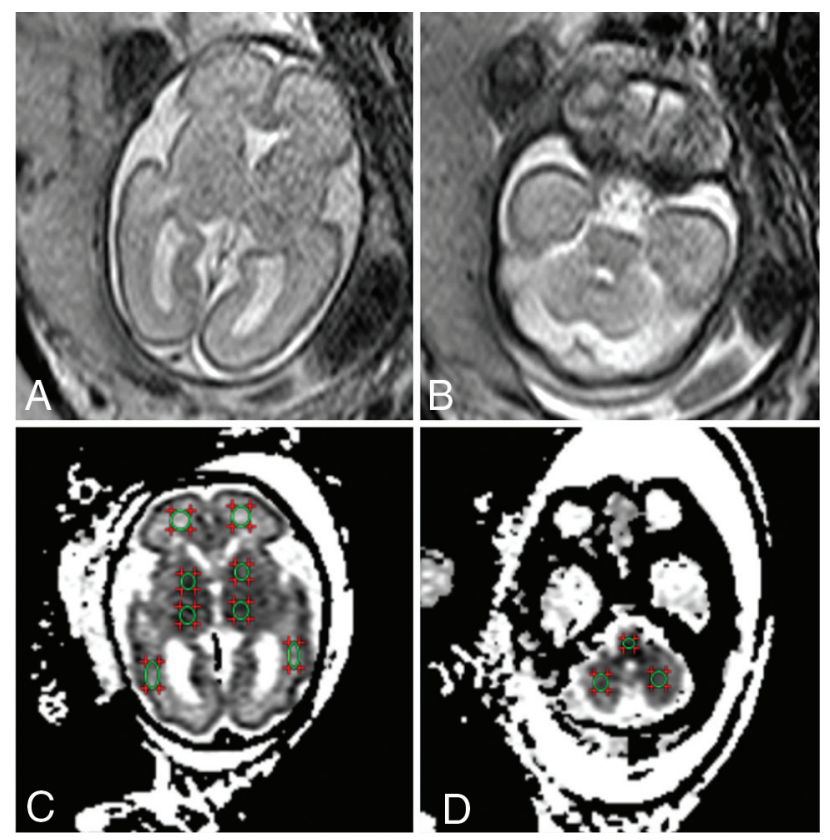

FIG 1. $A D C$ map in a fetus at 26 weeks' GA showing ROls in the different regions. $A$ and $B, T 2 W I$. $C$ and $D$, The same GA, DWI. Pair-wise $A D C$ values of the ROls are manually delineated on each side of the frontal WM, periatrial WM, basal ganglia, thalamus, and cerebellar hemisphere, as well as a single measurement in the pons.
In the control group, indications for fetal brain MR imaging included the following: limitations of fetal sonography: maternal abdominal wall edema $(n=10)$, uterine myomas $(n=10)$, oligohydramnios $(n=20)$, unfavorable fetal lie $(n=15)$; extra-CNS abnormalities: bilateral cleft lip $(n=12)$, mild hydronephrosis $(n=20)$, congenital cystic adenomatoid malformation $(n=3)$, intestinal duplication $(n=3)$, ovarian cyst $(n=5)$, and hepatic cyst $(n=2)$.

Finally, data from 50 cases in the CHD group (range, 20 - 36 weeks; 25 fetuses in each of GA group: 20- to 25.9-week and 26- to 36-week groups) and 100 cases in the control group (range, $20-36$ weeks; 50 fetuses in each of the corresponding 2 groups) were enrolled in the MR imaging analysis. The mean GA of the CHD group was 26.1 (SD, 3.5) weeks, similar to that in the control population (GA, 26.6 [SD, 3.9] weeks). The clinical characteristics of our cohort are shown in Table 1.

\section{ADC Measurements}

The intraobserver analysis showed excellent reproducibility of all intracranial volumes when observer 1 re-analyzed a subgroup of 30 randomly selected subjects after 2 months (all intraclass correlation coefficients $>0.90$ ). Interobserver (observer 2) correlation coefficients were calculated for each mean ADC value of the frontal WM (0.80), periatrial WM (0.81), basal ganglia (0.82), thalamus (0.88), cerebellar hemisphere (0.87), and pons (0.86).

By means of a paired $t$ test, the ADC values of the left and right sides were not significantly different, so the ADC values of each anatomic position of the hemispheres could be averaged. A significant negative correlation was observed between advancing GA and ADC measurements obtained in the periatrial WM, basal ganglia, thalamus, cerebellar hemisphere, and pons (CHD group: $R^{2}=0.19,0.24,0.37,0.20$, and 0.23 ; control group: $R^{2}=0.47$, $0.31,0.65,0.54$, and 0.41 , respectively), while in the frontal WM, the ADC values revealed no statistical correlation with GA in the 2 groups ( $P=.38$ and 0.94 , respectively) (Fig 2).

$T$-test analysis indicated that fetuses with $\mathrm{CHD}$ had significantly lower ADC values in the frontal WM, periatrial WM, and pons than controls (all $P<.05$, respectively) in the early stages of pregnancy $(\mathrm{GA}<26$ weeks), while they were not significantly different in the periatrial WM and pons between the CHD and control groups in the late stages of pregnancy (GA $\geq 26$ weeks). Only frontal WM ADC values were significantly lower in cases of both early (GA $<26$ weeks) and late $\mathrm{CHD}$ (GA $\geq 26$ weeks) (all $P<.05)$. Although the CHD group with $\mathrm{GA}<26$ weeks had slighter lower $\mathrm{ADC}$ values in the thalamus than controls $(P=.22)$, the ADC values in the thalamus were significantly higher in fetuses with $\mathrm{CHD}$ than in the control population $(P<.05)$ in the late stages of pregnancy ( $\mathrm{GA} \geq 26$ weeks). As for the cerebellar hemisphere, there was a strong trend toward not reaching significance between cases and controls $(P=.07)$ in late stages of pregnancy ( $\mathrm{GA} \geq 26$ weeks). Basal ganglia ADC values $(P=.47 ; 0.21)$ were consistently 

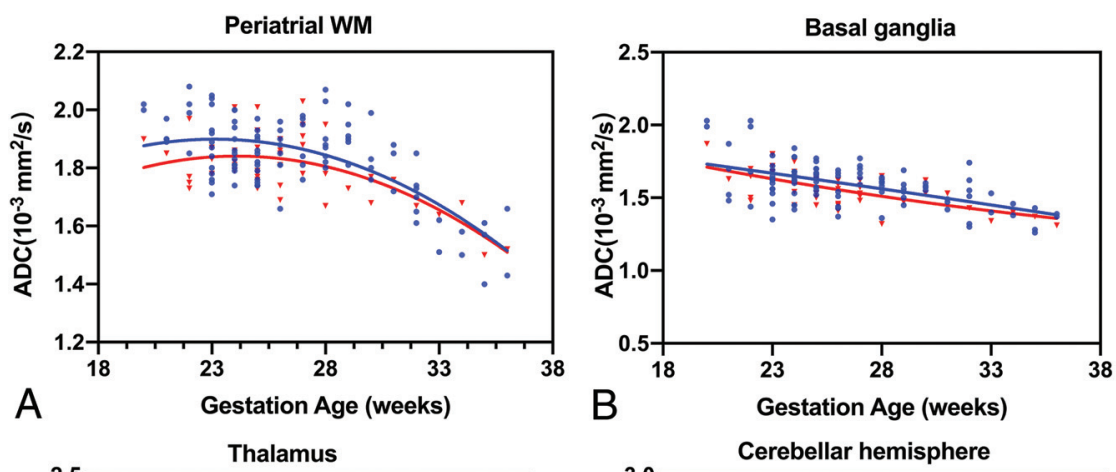

suggest that during the early stage of neurodevelopment in fetuses with $\mathrm{CHD}$, abnormal diffusivity in different brain areas may represent a difference in the structural organization of axons or glia. Our study also suggested that ADC values as measured by DWI could be a feasible method of evaluating brain injury in fetuses with certain CHDs.

Although sonography has always been the preferred imaging method for prenatal examination, MR imaging has obvious advantages over sonography in the display of neurologic maturation and abnormalities. ${ }^{12}$ While conventional MR imaging sequences (T1WI and T2WI) are desirable to assess morphologic anomalies, DWI can detect microstructural brain changes preceding changes on conventional MR images and has a potential role in detecting and characterizing diffuse injuries of the fetal brain. ${ }^{13}$ In this study, none of the CHD fetuses showed morphologic brain abnormalities, but the difference in ADC values deviating from the control group suggests abnormal brain axonal density.

Our results in the group with GA $<$ 26 weeks are similar to those in previously published fetal DWI studies. ${ }^{89}$ Higher ADC values of supratentorial WM regions (frontal WM, periatrial WM) can be interpreted because of the presence of immature migrant cells, an

not significantly different between the 2 groups in the early and late stages of pregnancy (Fig 3 and Table 2).

For the CHD and control groups, the mean ADC values of the supratentorial WM regions (frontal WM, periatrial WM) were consistently higher than those of the infratentorial regions (cerebellar hemisphere, pons), and the mean ADC values of the deep gray matter (basal ganglia, thalamus) were consistently similar to each other during the early and late stages of pregnancy, as shown in Table 2.

\section{DISCUSSION}

We used fetal DWI to show abnormal diffusion in different regions of the brain in fetuses with $\mathrm{CHD}$ compared with controls. These results reinforce the previous studies reporting that some congenital cardiac lesions can affect the development of the fetal brain in utero. ${ }^{10,11}$ We observed regional differences in ADC during gestation, likely reflecting differences in fetal brain development because of many factors such as changes in the tissue cellularity and water content, neuronal maturation, neuronal remodeling and pruning, axonal sprouting, glial proliferation, and so forth. Our results increase in cellular density, and a loose tissular organization with larger extracellular spaces within the WM. The lower ADC values of the cerebellum, pons, and thalamus maybe due to earlier maturation and myelination. In the group with $\mathrm{GA} \geq 26$ weeks, our results corroborate previous abnormal ADC values in a smaller cohort of 3 fetuses with CHD with a mean GA of 34 weeks, reported by Berman et al, ${ }^{14}$ who found that the ADC values of the thalamus in the CHD group were significantly higher than those in the control group; however, the values in the basal ganglia were not significantly different. Schönberg et $\mathrm{al}^{15}$ also described ADC values in 8 fetuses affected by CHD with a mean GA of 32.5 weeks and found an increase in ADC values in all regions of the WM as well as in the basal ganglia, comparable with values found in healthy fetuses. In fetuses with $\mathrm{CHD}$ of $<26$ weeks' GA, our results showed a significant reduction in the ADC values of the WM and pons compared with the control group.

Several reasons could explain the difference among these studies. First, because of the characteristics of fetal brain development, most published fetal studies on this topic have been conducted in the third trimester. ${ }^{16}$ However, many key steps of brain development have been completed by midgestation (GA, 21 - 26 weeks), 
such as neuronal migration and dendritic formation, synapse formation, and oligodendrocyte maturation. ${ }^{17}$ Therefore, our cohort study with an average GA of 26 weeks was chosen to determine the timing of intrauterine brain structural changes associated with CHD. Some studies have demonstrated that in normal deep WM areas, $\mathrm{ADC}$ values initially tend to increase from 20 gestational weeks up to a peak around 30 weeks. ${ }^{8,9}$ Due to the decrease in water content and the beginning of higher-order maturation, this increase is rapidly followed by a subsequent decrease in ADC

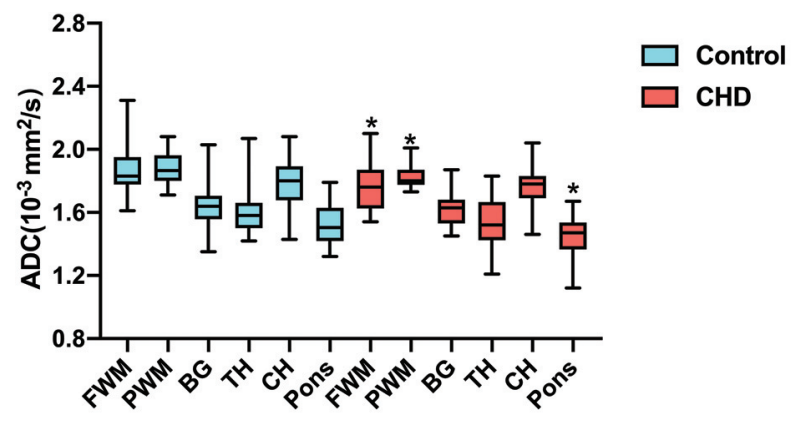

21 weeks $\leq \mathrm{GA}<26$ weeks

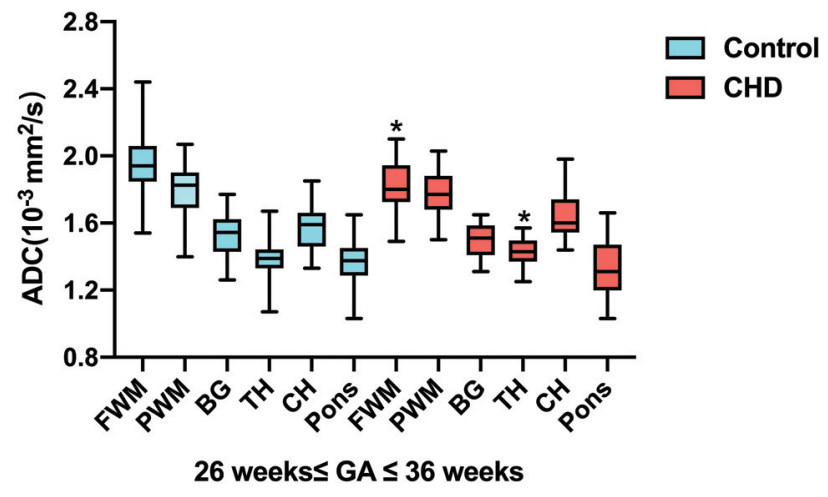

FIG 3. Box-and-whisker plots representing the distribution of ADC measurements across the fetal brain anatomic structures and pregnancy trimesters. Asterisks indicate significant differences between the CHD (red) and control (blue) groups for the same structure. BG indicates basal ganglia; $\mathrm{TH}$, thalamus; $\mathrm{CH}$, cerebellar hemisphere; FWM, frontal WM; PWM, periatrial WM.

Table 2: ADC values in the CHD and control groups in different brain areas ${ }^{\mathrm{a}}$

\begin{tabular}{lcccc}
\hline & Unit & Control $(\boldsymbol{n}=100)$ (mean) & CHD $(\boldsymbol{n}=\mathbf{5 0})$ (mean) & $\boldsymbol{P}$ Value \\
\hline GA $<26$ wk (No.) & & 50 & 25 & \\
Frontal WM & $\left(10^{-3} \mathrm{~mm}^{2} / \mathrm{s}\right)$ & $1.87(\mathrm{SD}, 0.15)$ & $1.77(\mathrm{SD}, 0.14)$ & $.01^{\mathrm{b}}$ \\
Periatrial WM & $\left(10^{-3} \mathrm{~mm}^{2} / \mathrm{s}\right)$ & $1.88(\mathrm{SD}, 0.10)$ & $1.83(\mathrm{SD}, 0.08)$ & $.04^{\mathrm{b}}$ \\
Basal ganglia & $\left(10^{-3} \mathrm{~mm}^{2} / \mathrm{s}\right)$ & $1.65(\mathrm{SD}, 0.15)$ & $1.62(\mathrm{SD}, 0.11)$ & .47 \\
Thalamus & $\left(10^{-3} \mathrm{~mm}^{2} / \mathrm{s}\right)$ & $1.61(\mathrm{SD}, 0.16)$ & $1.55(\mathrm{SD}, 0.15)$ & .22 \\
CH & $\left(10^{-3} \mathrm{~mm}^{2} / \mathrm{s}\right)$ & $1.79(\mathrm{SD}, 0.15)$ & $1.77(\mathrm{SD}, 0.12)$ & .40 \\
Pons & $\left(10^{-3} \mathrm{~mm}^{2} / \mathrm{s}\right)$ & $1.53(\mathrm{SD}, 0.13)$ & $1.44(\mathrm{SD}, 0.13)$ & $.03^{\mathrm{b}}$ \\
GA $\geq 26$ wk (No.) & & 50 & 25 & \\
Frontal WM & $\left(10^{-3} \mathrm{~mm}^{2} / \mathrm{s}\right)$ & $1.94(\mathrm{SD}, 0.18)$ & $1.82(\mathrm{SD}, 0.15)$ & $.006^{\mathrm{b}}$ \\
Periatrial WM & $\left(10^{-3} \mathrm{~mm}^{2} / \mathrm{s}\right)$ & $1.80(\mathrm{SD}, 0.16)$ & $1.78(\mathrm{SD}, 0.13)$ & .41 \\
Basal ganglia & $\left(10^{-3} \mathrm{~mm}^{2} / \mathrm{s}\right)$ & $1.53(\mathrm{SD}, 0.13)$ & $1.50(\mathrm{SD}, 0.10)$ & .21 \\
Thalamus & $\left(10^{-3} \mathrm{~mm}^{2} / \mathrm{s}\right)$ & $1.39(\mathrm{SD}, 0.11)$ & $1.44(\mathrm{SD}, 0.08)$ & $.04^{\mathrm{b}}$ \\
CH & $\left(10^{-3} \mathrm{~mm}^{2} / \mathrm{s}\right)$ & $1.58(\mathrm{SD}, 0.13)$ & $1.65(\mathrm{SD}, 0.13)$ & .07 \\
Pons & $\left(10^{-3} \mathrm{~mm}^{2} / \mathrm{s}\right)$ & $1.37(\mathrm{SD}, 0.13)$ & $1.34(\mathrm{SD}, 0.15)$ & .38 \\
\hline
\end{tabular}

Note:- $\mathrm{CH}$ indicates cerebellar hemisphere.

${ }^{a}$ Data are presented as means.

${ }^{\mathrm{b}} \mathrm{P}<.05$. values in most brain areas after 30 weeks. ${ }^{18}$ Therefore, in the early stages of pregnancy (GA $<26$ weeks), the reduced mean ADC values in the WM of the CHD group probably represent delayed maturation.

Another source of the difference is that the types of CHD included in the cohort study were diverse. Of note, these previous findings ${ }^{14,15}$ in regional tissue development were associated with a more specific type of complex CHD: HLHS. However, other CHD types with decreased oxygen delivery to the brain can also be associated with prenatal neurologic injury. Good examples of CHD types with decreased oxygenation of the brain are TGA and TOF. In TGA, ${ }^{19}$ the aorta arises from the right ventricle and the fetal brain receives deoxygenated blood returning from the systemic circulation stream. In fetuses with $\mathrm{TOF},{ }^{20}$ the presence of a large ventricular septal defect leads to mixing of blood in the left and right ventricles, which results in consecutively low oxygen saturation in the cerebral arteries. Moreover, Donofrio et $\mathrm{al}^{21}$ evaluated cerebral blood flow perfusion changes in fetuses with CHD using head circumference and the cerebral-to-placental resistance ratio and found that fetuses with TGA were less affected than those with HLHS. These results indicate that diverse cardiac lesions are likely to have different brain effects. In addition, there is also a large difference in sample size.

Our results are aligned with Arthurs at al that showed that a significant decline was found for ADC values in the frontal WM compared with healthy fetuses, suggesting a high susceptibility to frontal chronic hypoxic-ischemic insult with pronounced abnormalities in frontal WM perfusion in fetuses with $\mathrm{CHD} .{ }^{22} \mathrm{We}$ hypothesized that this phenomenon may represent chronic damage and WM gliosis from a decline in ADC values due to hypercellularity. A previous study ${ }^{23}$ concluded that the thalamus, in particular, was more sensitive to hypoxia changes, consistent with our results that the CHD group had higher ADC values during the later stages of pregnancy. In our study, we did not observe abnormal diffusivity in the basal ganglia. We also found that although the CHD group with GA $<26$ weeks had lower ADC values in the pons than in controls, there was not a significant difference between the 2 groups in the later stages of pregnancy ( $G A \geq 26$ weeks). The higher cognitive functions of the frontal lobes are protected by a compensatory mechanism such as the "brain-sparing" effect during an earlier pregnancy. ${ }^{24}$ However, under chronic circumstances or a later pregnancy, perfusion redistribution aims to protect more elementary brain regions such as the basal ganglia and pons supplied by the middle and posterior cerebral arteries. Therefore, our data indicate that different regions of the brain differ in the sensitivity to hypoxic-ischemic injury during the development process.

There are some limitations to this study. Our sample size was limited, resulting in a failure to perform a more detailed classification analysis according 
to the characteristics of the types of CHD. Second, postpartum imaging follow-up studies and long-term standardized neuropsychological evaluations are required to determine the value of DWI for the prenatal diagnosis of fetuses with CHD. Because our study included fetuses with severe $\mathrm{CHD}$, most of whom required corrective surgery during infancy, certain cognitive delays or involved disabilities that are only diagnosed during school age make follow-up difficult.

\section{CONCLUSIONS}

The ADC value of fetuses with CHD was significantly decreased in different brain areas during the early and late stages of pregnancy compared with healthy controls. This study suggests that abnormal brain diffusivity detected by fetal brain DWI could be a feasible early marker of axonal development from midgestation, which may lead to brain growth failure during the third trimester. This technique could aid in recognizing a possible detrimental effect of CHD on developing brain tissue. However, the prognostic value of $\mathrm{ADC}$ changes in postnatal development requires further study.

\section{REFERENCES}

1. Hansen T, Henriksen TB, Bach CC, et al. Congenital heart defects and measures of prenatal brain growth: a systematic review. Pediatr Neurol 2017;72:7-18 CrossRef Medline

2. Peyvandi S, Latal B, Miller SP, et al. The neonatal brain in critical congenital heart disease: Insights and future directions. Neuroimage 2019;185:776-82 CrossRef Medline

3. von Rhein M, Buchmann A, Hagmann C, et al. Severe congenital heart defects are associated with global reduction of neonatal brain volumes. J Pediatr 2015;167:1259-63 CrossRef Medline

4. Lie ML, Graham RH, Robson SC, et al. MERIDIAN Collaborative Group. MRI for fetal developmental brain abnormalities: perspectives from the pregnant patient. Qual Health Res 2018;28:1295-1307 CrossRef Medline

5. Le Bihan D. Apparent diffusion coefficient and beyond: what diffusion MR imaging can tell us about tissue structure. Radiology 2013;268:318-22 CrossRef Medline

6. Sartor A, Arthurs O, Alberti C, et al. Apparent diffusion coefficient measurements of the fetal brain during the third trimester of pregnancy: how reliable are they in clinical practice? Prenat Diagn 2014;34:357-66 CrossRef Medline

7. Cartry C, Viallon V, Hornoy P, et al. Diffusion-weighted MR imaging of the normal fetal brain: marker of fetal brain maturation. $J$ Radiol 2010;91:561-66 CrossRef Medline

8. Schneider JF, Confort-Gouny S, Le Fur Y, et al. Diffusion-weighted imaging in normal fetal brain maturation. Eur Radiol 2007;17:242229 CrossRef Medline
9. Schneider MM, Berman JI, Baumer FM, et al. Normative apparent diffusion coefficient values in the developing fetal brain. AJNR Am J Neuroradiol 2009;30:1799-1803 CrossRef Medline

10. Claessens NH, Khalili N, Isgum I, et al. Brain and CSF volumes in fetuses and neonates with antenatal diagnosis of critical congenital heart disease: a longitudinal MRI study. AJNR Am J Neuroradiol 2019;40:885-91 CrossRef Medline

11. Mebius MJ, Kooi EM, Bilardo CM, et al. Brain injury and neurodevelopmental outcome in congenital heart disease: a systematic review. Pediatrics 2017;140:e20164055 CrossRef Medline

12. Griffiths PD, Bradburn M, Campbell MJ, et al. Use of MRI in the diagnosis of fetal brain abnormalities in utero (MERIDIAN): a multicentre, prospective cohort study. Lancet 2017;389:538-46 CrossRef Medline

13. Arthurs OJ, Thayyil S, Pauliah SS, et al. Diagnostic accuracy and limitations of post-mortem MRI for neurological abnormalities in fetuses and children. Clin Radiol 2015;70:872-80 CrossRef Medline

14. Berman JI, Hamrick SE, McQuillen PS, et al. Diffusion-weighted imaging in fetuses with severe congenital heart defects. AJNR Am J Neuroradiol 2011;32:E21-22 CrossRef Medline

15. Schönberg N, Weisstanner C, Wiest R, et al. The influence of various cerebral and extracerebral pathologies on apparent diffusion coefficient values in the fetal brain. J Neuroimaging 2020;30:47785 CrossRef Medline

16. Clouchoux C, Du Plessis AJ, Bouyssi-Kobar M, et al. Delayed cortical development in fetuses with complex congenital heart disease. Cereb Cortex 2013;23:2932-43 CrossRef Medline

17. Kostović I, Jovanov-Milosević N. The development of cerebral connections during the first 20-45 weeks' gestation. Semin Fetal Neonatal Med 2006;11:415-22 CrossRef Medline

18. Han R, Huang L, Sun Z, et al. Assessment of apparent diffusion coefficient of normal fetal brain development from gestational age week 24 up to term age: a preliminary study. Fetal Diagn Ther 2015;37:102-07 CrossRef Medline

19. Alsaied T, Tseng S, King E, et al. Effect of fetal hemodynamics on growth in fetuses with single ventricle or transposition of the great arteries. Ultrasound Obstet Gynecol 2018;52:479-87 CrossRef Medline

20. Schellen C, Ernst S, Gruber GM, et al. Fetal MRI detects early alterations of brain development in Tetralogy of Fallot. Am J Obstet Gynecol 2015;213:392.e1-7 CrossRef Medline

21. Donofrio MT, Bremer YA, Schieken RM, et al. Autoregulation of cerebral blood flow in fetuses with congenital heart disease: the brain sparing effect. Pediatr Cardiol 2003;24:436-43 CrossRef Medline

22. Arthurs OJ, Rega A, Guimiot F, et al. Diffusion-weighted magnetic resonance imaging of the fetal brain in intrauterine growth restriction. Ultrasound Obstet Gynecol 2017;50:79-87 CrossRef Medline

23. Papadopoulou I, Langan D, Sebire NJ, et al. Diffusion-weighted post-mortem magnetic resonance imaging of the human fetal brain in situ. Eur J Radiol 2016;85:1167-73 CrossRef Medline

24. Cohen E, Baerts W, van Bel F. Brain-sparing in intrauterine growth restriction: considerations for the neonatologist. Neonatology 2015;108:269-76 CrossRef Medline 